\title{
Körperliches Training: hohe Effektstärken bei einem ganzen Strauß psychischer Erkrankungen
}

\author{
Sport als moderne Polypille ist schon vielfach \\ gepriesen worden. Es kommt offenbar weniger auf \\ die Dosis oder Intensität an: Moderate und intensive \\ Belastungen sind ebenso wirkungsvoll wie leichte. \\ In fachgerecht angeleiteten Sportgruppen ist die \\ Abbrecherquote naturgemäß erniedrigt.
}

Eine Metaanalyse von 25 Studien [1] erhärtet nun den Nutzen von Sport bei depressiven Erkrankungen mit relativ hohen Effektstärken, berichtete Professor Martin Bohus vom Zentralinstitut für seelische Erkrankungen in Mannheim. Dies galt sowohl für die Major Depression (MDD) als auch für mildere Erkrankungsformen. Ein direkter Vergleich zwischen Sport und kognitiver Verhaltenstherapie (CBT) [2], ebenfalls bei mittelschwerer Depression ergab eine annähernde Gleichwertigkeit zwischen Sport, psychiatrischer Behandlung und einer Internetbasierten CBT-Therapie ( A Abb. 1). Die Abbruchraten lagen bei allen drei Therapieformen im niedrigen Bereich zwischen neun und $14 \%$. Der Nutzen von Sport bei schwerer Depression gilt

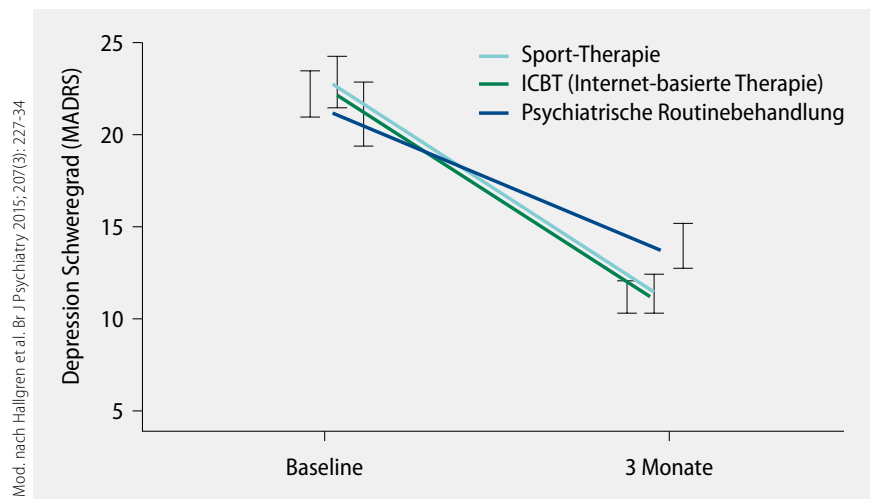

1 Mittelschwere Depression: Die Unterschiede hinsichtlich Wirksamkeit zwischen Sporttherapie, internetbasierter Therapie und psychiatrischer Routinebehandlung. auch für stationäre Patienten, wie eine weitere kontrollierte Studie belegt: Ein dreimal pro Woche absolviertes Kraftausdauerprogramm zusätzlich zur psychiatrischen Standardtherapie besserte die Werte der Patienten auf der Hamilton-Depressionsskala (HAM-D) signifikant um 4,4 Punkte.

Auch zur Prognose bei schizophrenen Psychosen kann Sport einen positiven Beitrag leisten, wenngleich deutlich weniger ausgeprägt als bei der Depression. Da die medikamentöse Therapie mit Neuroleptika oft ungünstige Stoffwechselveränderungen und erhöhte kardiovaskuläre Risiken mit sich führt, geht es hier mehr um physiologische als um psychologische Parameter. Eine Metaanalyse von 17 Studien und insgesamt über 650 Schizophreniepatienten [3] ergab zwar für sportliche Aktivitäten (im Mittel 75 Minuten pro Woche) keinen Einfluss auf den BMI, aber indirekt eine Reduktion des kardiovaskulären Risikos um 15-20\%. Wurden nur Studien mit einer sportlichen Aktivität von mindestens 90 Wochenminuten berücksichtigt, fanden sich auch klare positive Wirkungen auf Positiv- und Negativsymptomatik und in der Folge auch auf die Lebensqualität der Patienten. Auch im psychosomatischen Bereich, der Prophylaxe von Depressionen und Fatigue bei Krebspatienten fanden sich zwar eher mäßige, aber signifikante Wirkungen von Sport [3].

Bleibt das Problem der mangelnden Compliance: Eine Analyse von Daten aus Psychiatrischen Kliniken der Deutschschweiz ergab, dass trotz eines Sporttherapie-Angebotes bei $97 \%$ der Kliniken nur maximal 25-50\% der Patienten dieses Angebot auch annehmen. Als häufigste Motive für eine Ablehnung der Angebote ergab eine weitere Untersuchung [4] Selbstunsicherheit, Antriebsmangel, Niedergeschlagenheit und Unsicherheit.

Dr. Andreas Häckel, freier Medizinjournalist

Psyche und Sport (Martin Bohus, Mannheim). Psychiatrie Update, Mainz,

24.3.2017

Literatur

. Schuch FB et al. J Psychiatr Research 2016; 77: 42 - 51

2. Hallgren M et al. Br J Psychiatry 2015; 207 (3): 227 - 34

. Firth J et al. Psychol Med 2015; 45 (07): $1343-61$

4. Firth J et al. Psychol Med 2016; 46: $2869-81$

\section{Chronische Insomnie - Keine Angst vor Z-Substanzen!}

\section{Eigentlich sollten moderne Hypnotika nicht länger als vier Wochen verordnet werden. Manche Schlaf- mediziner halten diese Empfehlung jedoch für ver- fehlt. Die Risiken einer unbehandelten Insomnie wiegen für sie schwerer als die Suchtgefahr.}

Es sind Medienberichte wie der in der „ZEIT“ vom Juni 2015, die den Schlafmediziner Dr. Dieter Kunz immer wieder ärgern: Darin wird behauptet, dass Hypnotika zwar zunächst helfen, auf
Dauer aber massiv schaden [1]. Mit Bezug auf den Gesundheitswissenschaftler Professor Gerd Glaeske geht die Zeitung von bis zu 1,5 Millionen Schlafmittelabhängigen in Deutschland aus, wobei einfach angenommen wird, dass jeder, der ein Hypnotikum länger als drei Monate nimmt, davon abhängig ist. Auf diese Weise lässt sich dann eine „massenhafte Abhängigkeit“ konstruieren. In Deutschland gebe es jedoch nicht 1,5 Millionen Hypnotika-Abhängige, sondern „1,5 Millionen Patienten mit schlecht behandelten psychischen Störungen“, sagte Kunz auf der Fortbildungsveranstaltung „Psychiatrie Update“ in Mainz. 